\section{Kidney \\ Blood Pressure Research}

\title{
Salt Sensitivity Determined From Capillary Blood
}

\author{
Hans Oberleithner Marianne Wilhelmi \\ Institute of Physiology II, University Hospital, Westfälische-Wilhelms-Universität, Münster, Germany
}

\section{Key Words}

Hypertension • Sodium buffer capacity • Sodium sensitivity • Glycocalyx • Erythrocyte

\begin{abstract}
Background/Aims: A significant rise of blood pressure in response to a given salt load is a weak indication of high salt sensitivity, supposed to foster the development of arterial hypertension and related diseases in later life. In search of an alternative method we recently developed the salt blood test (SBT), a new concept for quantifying salt sensitivity (SS). Based on this concept, namely that red blood cells (RBC) report on salt sensitivity, the SBT-mini was developed. Methods: The SBT-mini utilizes a droplet of capillary blood mixed with a 'smart' $\mathrm{Na}^{+}$cocktail. Red blood cells (RBC) of this mixture are allowed to sediment by gravity in a glass tube. SS is quantified by measuring RBC sedimentation rate. 90 healthy volunteers (39 males, 51 females; mean age: $23 \pm 0.5$ years) were evaluated and 'standard values' for males and females were derived. Results: Sodium buffer capacity of female blood is about $20 \%$ smaller as compared to male blood due to the lower hematocrit of females. SS of an individual is related to the mean standard value (set to $100 \%$ ) of the respective male/female cohort. High SS (> $120 \%$ ) has been found in $31 \%$ of males and $28 \%$ of females. Conclusions: SS can be estimated derived from the individual RBC sodium buffer capacity as measured by the SBT-mini. About one third of a healthy test cohort exhibits a high sensitivity to salt. Reduction of sodium consumption to at least two grams per day (equals five grams of $\mathrm{NaCl}$ per day as suggested by the $\mathrm{WHO}$ ) is recommended, particularly for individuals with high salt sensitivity.
\end{abstract}

\section{Introduction}

Salt sensitivity (SS) characterizes the ability of the individual organism to control body sodium. Daily intake of large amounts of salt $(\mathrm{NaCl})$, very common in most societies worldwide, is known to foster the development of arterial hypertension damaging brain, heart, kidney and the blood vessel system [1-8]. Although it would be very useful being able to quantify SS, practicable methods are barely at hand. Accurate testing of SS is usually 


\section{Kidney \\ Blood Pressure Research}

laborious and associated with low patient compliance [9-11]. Recently, the so-called salt blood test (SBT) was developed $[12,13]$. It describes salt sensitivity of erythrocytes in quantitative terms and, indirectly, allows insight into the functional properties of the inner surface layer of blood vessels. We found a positive correlation between erythrocyte salt sensitivity and blood pressure though we do not know yet whether this relationship is coincidental or causative [14]. The test is based on a blood sample taken from venous puncture. Red blood cells (RBC) must be isolated, washed and suspended in two different $\mathrm{Na}^{+}$cocktails before measurements can be performed. Although this procedure is already rather simple, it needs several milliliters of venous blood, some washing procedures and the use of a centrifuge. Therefore, this SBT needs some basic laboratory expertise and equipment which is not accessible everywhere. Since a test for SS should be as simple as possible so that everybody should be able to perform it, independent of laboratory skills, we modified (i.e. simplified) the original SBT. The emphasis was on "fewer steps" and "smaller blood samples". We finally arrived at a new design, the SBT-mini. This test needs only $50 \mu$ of capillary blood, requires no centrifugation of the sample and, for comparison, uses standard values for males and females obtained from healthy individuals. Nevertheless, the SBT-mini reports on SS in quantitative terms, with a technical scatter in the range of $5 \%$.

\section{Materials and Methods}

\section{Salt Blood Test (SBT-mini)}

Figure 1 shows the performance of the SBT-mini. (A) shows the five components of the test (from left to right: safety lancet, Sarstedt, Nümbrecht, Germany; Minivette ${ }^{\odot}$ POCT, Tri-K-EDTA, Sarstedt; PCR soft tube, $0.2 \mathrm{ml}$, Biozym Scientific GmbH, filled with $50 \mu \mathrm{l}$ of $\mathrm{Na}^{+}$cocktail, CARE diagnostica Laborreagenzien $\mathrm{GmbH}$, Voerde, Germany; hematocrit tube Safecap P75-2000M, Scholz GmbH, Neubiberg, Germany; plexiglass home-built tube holder. (B) Filling of the Minivette ${ }^{\odot}$ by capillary forces. Blood enters and stops automatically when filled $(50 \mu \mathrm{l})$. (C) Blood is delivered into the $\mathrm{Na}^{+}$cocktail and mixed well by hand for about 20 seconds. (E) The blood $/ \mathrm{Na}^{+}$cocktail mixture is allowed to enter the hematocrit tube by the use of capillary/gravity forces. (F) The hematocrit tube is vertically mounted in the tube holder and the length of the RBC-free supernatant $\left(S_{x}\right)$ is measured after 60 minutes. $(G)$ SS is calculated as follows:

$$
\operatorname{SS}(\%)=\left(S_{x} / \text { standard }\right) \cdot 100
$$

$S_{x}$ and standard are given in millimeters. The standard value for males is $21.4 \mathrm{~mm}$ (60 min value) and the one for females is $26.1 \mathrm{~mm}$ ( $60 \mathrm{~min}$ value). The two standard values serve as reference values derived from 39 male and 51 female healthy volunteers, respectively. Mean SS obtained from each of these two cohorts is set to $100 \%$ indicating average SS in a normal population.

In addition, hematocrit values were obtained for each individual by centrifugation of the same sample as used for the SS measurements. Hematocrit is given as the erythrocyte volume percentage (\%) of whole blood. A video that describes the concept and the step-by-step procedure of the test can be found at: https:// www.youtube.com/watch?v=afqIGhJhqig.

\section{Statistics}

Data are expressed as mean \pm SEM or SD as appropriate. Significance was tested with the unpaired student's $t$ test. Non-parametric data were tested for significance with the Mann-Whitney-U-test. Data were termed "significantly different" when the probability of error was smaller than $5 \%(p<0.05)$.

\section{Results}

Figure 2 shows the lengths of the supernatants measured at different ambient $\mathrm{Na}^{+}$concentrations over time. Please note that the length of the supernatant that develops over a giv- 


\section{Kidney \\ Blood Pressure \\ Research}
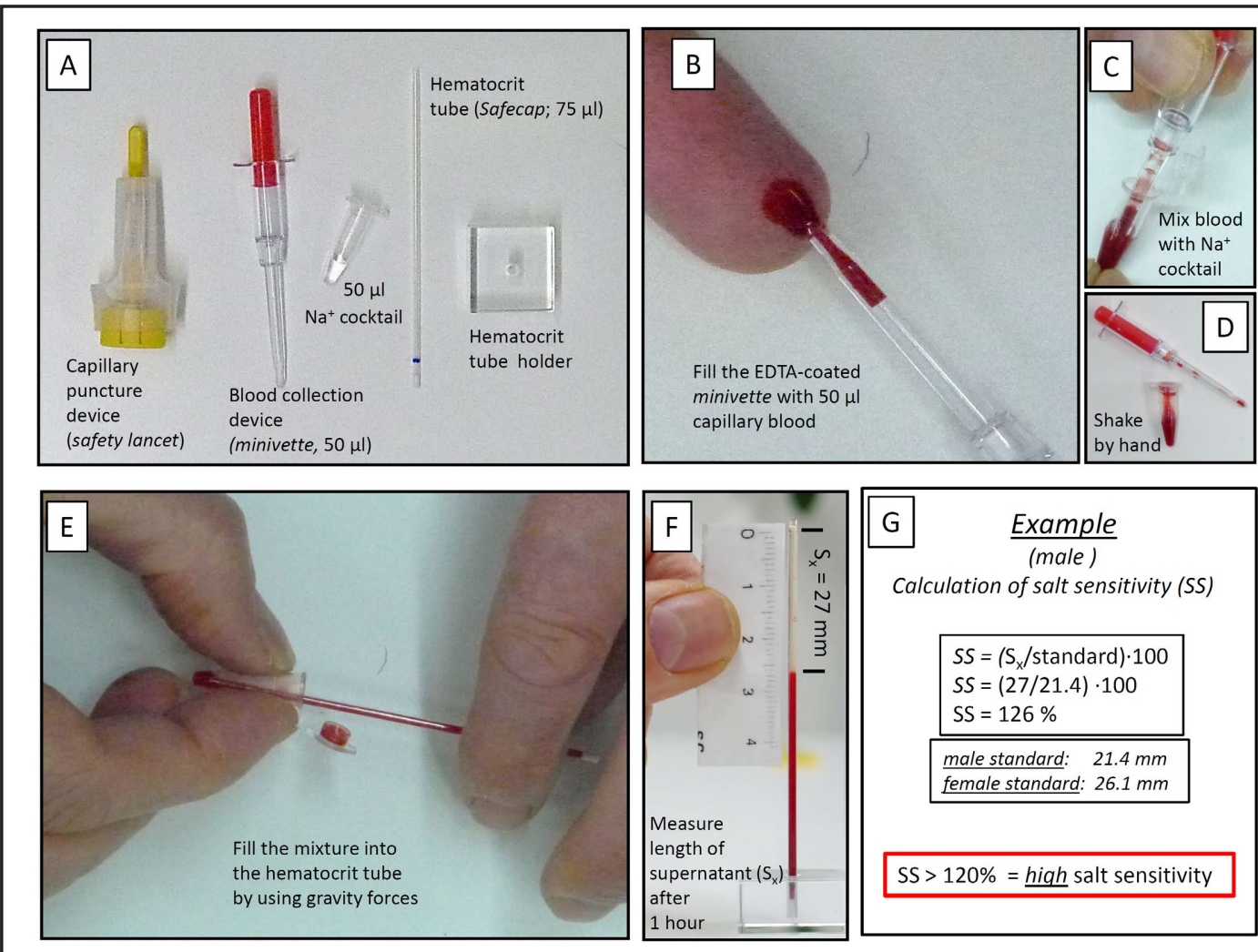

Fig. 1. Salt blood test procedure (SBT-mini). (A) Five items necessary for carrying out the test. (B) Blood sampling from the fingertip. (C) - (D) Mixing blood with the $\mathrm{Na}^{+}$cocktail. (E) Filling of the hematocrit tube by capillary/gravity forces. (F) Measuring supernatant length after 1 hour. (G) Calculating salt sensitivity (\%). The length of the individual supernatant $\left(\mathrm{S}_{\mathrm{x}}\right.$ in $\mathrm{mm}$ ) is divided by the mean length of supernatants of either the male cohort (standard=21.4 mm; $\mathrm{n}=39$ ) or the female cohort (standard=26.1 mm; $\mathrm{n}=51$ ). This ratio $\left(\mathrm{S}_{\mathrm{x}}\right.$ /standard $)$ multiplied by 100 expresses the salt sensitivity (SS) of an individual in percent. $100 \pm 20$ $\%$ indicates average SS of the healthy population based on the mean SS values of 39 measurements in males and 51 measurements in females.

Fig. 2. Length of supernatant is shown as a function of time and ambient $\mathrm{Na}^{+}$. Five different $\mathrm{Na}^{+}$concentrations were tested. The red symbol indicates the experimental condition used for the SBT-mini (111 $\mathrm{mM} \mathrm{Na}^{+}$as a result of mixing $50 \mu \mathrm{l}$ of whole blood with $50 \mu \mathrm{l}$ of a $90 \mathrm{mM} \mathrm{Na}^{+}$ cocktail). Each concentration was tested 5 times. Mean values are given. For the sake of clarity only one curve shows mean values with standard deviations (mean \pm SD).

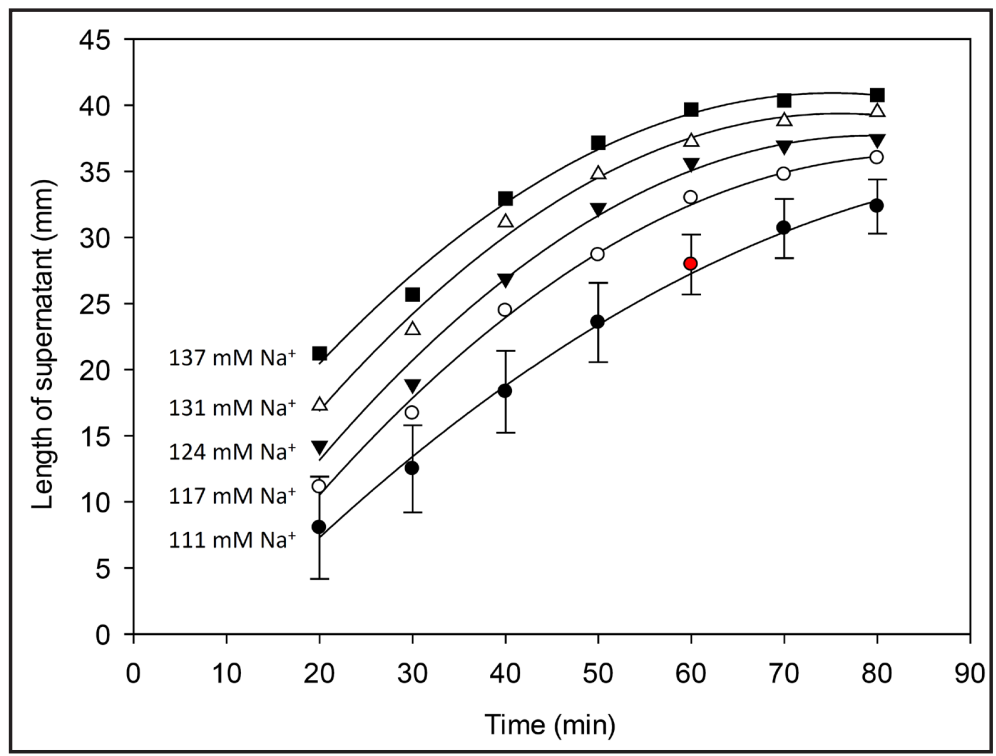




\section{Kidney Blood Pressure Research}

en time period at a specific $\mathrm{Na}^{+}$ concentration is positively correlated to the individual's SS. The different (ambient) $\mathrm{Na}^{+}$concentrations (in mM: $111,117,124$, 131 and 137) were created by mixing $50 \mu \mathrm{l}$ of whole blood (taken from the Minivette $^{\odot}$ ) with $50 \mu \mathrm{l}$ of five different $\mathrm{Na}^{+}$cocktails. As expected the speed of supernatant formation (i.e. the

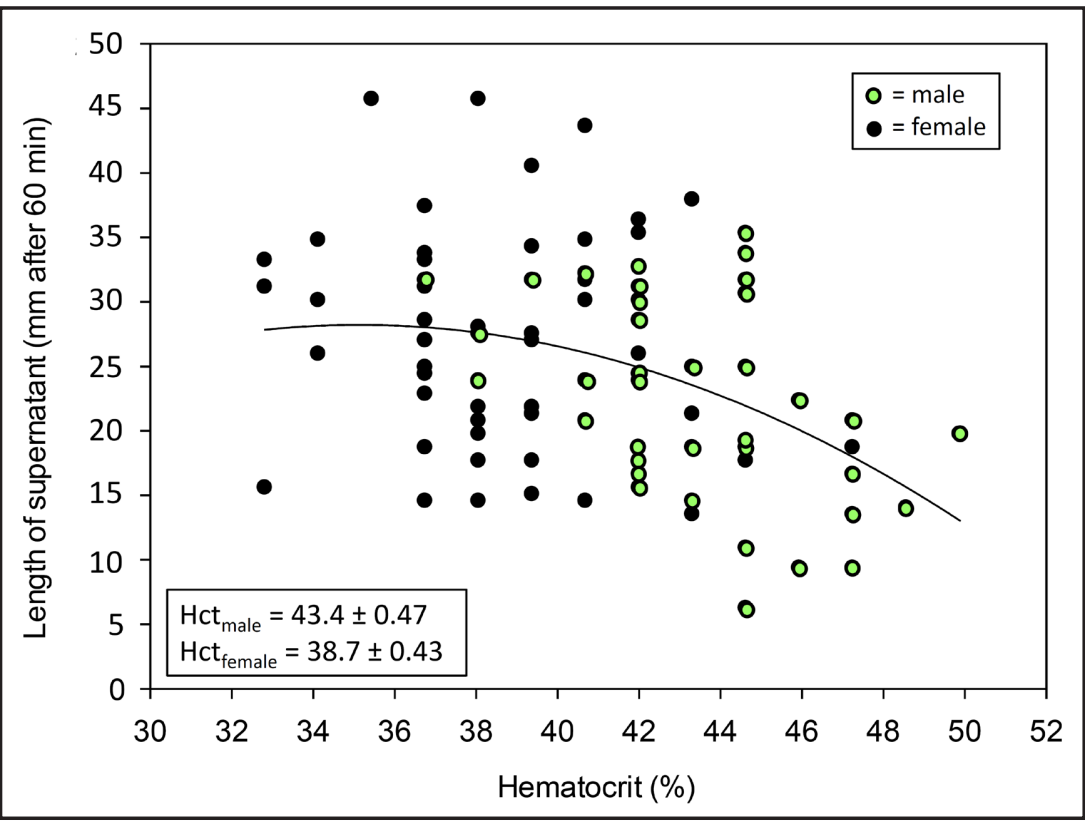

Fig. 3. Lengths of supernatants $(\mathrm{mm} / 60 \mathrm{~min})$ related to individual hematocrit values. Shown is a $2^{\text {nd }}$ order regression line $\left(\mathrm{r}^{2}=0.15\right)$.

sedimentation velocity) strong-

ly depends on the respective $\mathrm{Na}^{+}$concentrations. This confirms previous studies $[12,15,16]$. It is not due to different osmolalities since even balanced osmolalities (by adding adequate amounts of sucrose) does not influence the results (data not shown). The reason why we chose the time point "60 minutes" for the SBT-mini was as follows: (i) The separation between supernatant and erythrocyte sediment was (optically) sharp enough to allow a precise measurement of supernatant length (see figure $1 \mathrm{~F}$ ). (ii) The curve was well embedded between the minimal (about $10 \mathrm{~mm}$ ) and maximal (about $40 \mathrm{~mm}$ ) values of supernatant lengths that can be expected in samples from different (low and high salt sensitive) individuals. Figure 3 shows a graph in which the dependence of the length of the supernatant on the individual hematocrit values is displayed. It shows some dependence of SS mainly when hematocrit values are above $40 \%$. Such hematocrit values are typical (physiological) for the male cohort (green symbols). This is plausible because the RBC concentration influences the erythrocyte $\mathrm{Na}^{+}$buffering capacity of the individual sample. Therefore, we used two separate standard values, one for males and another one for females. Figure 4 shows the frequency distributions of supernatant lengths obtained in 90 healthy individuals ( 39 males, 51 females, mean age $=23 \pm 0.5$ years). The curve of the female participants is clearly located at the right side of the figure in comparison to the curve of male cohort. This is mainly due to the lower hematocrit of females. When this is taken into account and the data "corrected" for the unequal hematocrit values, then this sex difference vanishes, i.e. the curve of the female cohort shifts to the left as expected (figure 4). The histograms of the male and the female cohort exhibit two peaks, irrespective of the hematocrit and more pronounced in women, indicating that two subpopulations may exist, namely a low and a high salt sensitive one. Figures 5 and 6 show the individual SS values for the male and the female cohort. SS was calculated as mentioned above. The SS value of $100 \%$ is based on the mean SS value obtained for each cohort. Values between 80 and $120 \%$ were termed "average SS values" in order to indicate that values may considerably scatter. Low SS $(<80 \%)$ is found in $28 \%$ (males) and $31 \%$ (females). Average SS (80 to $120 \%$ ) is found in $41 \%$ (males and females) and high SS (> 120\%) is found in $31 \%$ (males) and $28 \%$ (females). Reliability of the SBT-mini is documented in table 1. Performing 15 tests in parallel (at the same time) using capillary blood of a single person exhibits a stan- 


\section{Kidney Blood Pressure Research}

dard deviation of $5 \%$. This indicates the 'technical' error. Performing 211 tests in a single person over a period of 7 months exhibits a standard deviation of $14 \%$. This number includes the 'technical' error plus any SS variations of this individual person over 7 months. It emphasizes the important point that the individual SS is not at all a constant value but a variable value that can undergo changes over time depending on salt consumption, medication, life style, etc. This view opens the perspective that the SBT-mini could serve as a useful test motivating people to adjust their life style / medication accordingly. The standard deviations of 39 and 51 measurements obtained in healthy male and female individuals are $35 \%$ and $31 \%$, respectively. These numbers include the 'technical' error (5\%, see above) plus the different SS values of these individuals. In other words, this

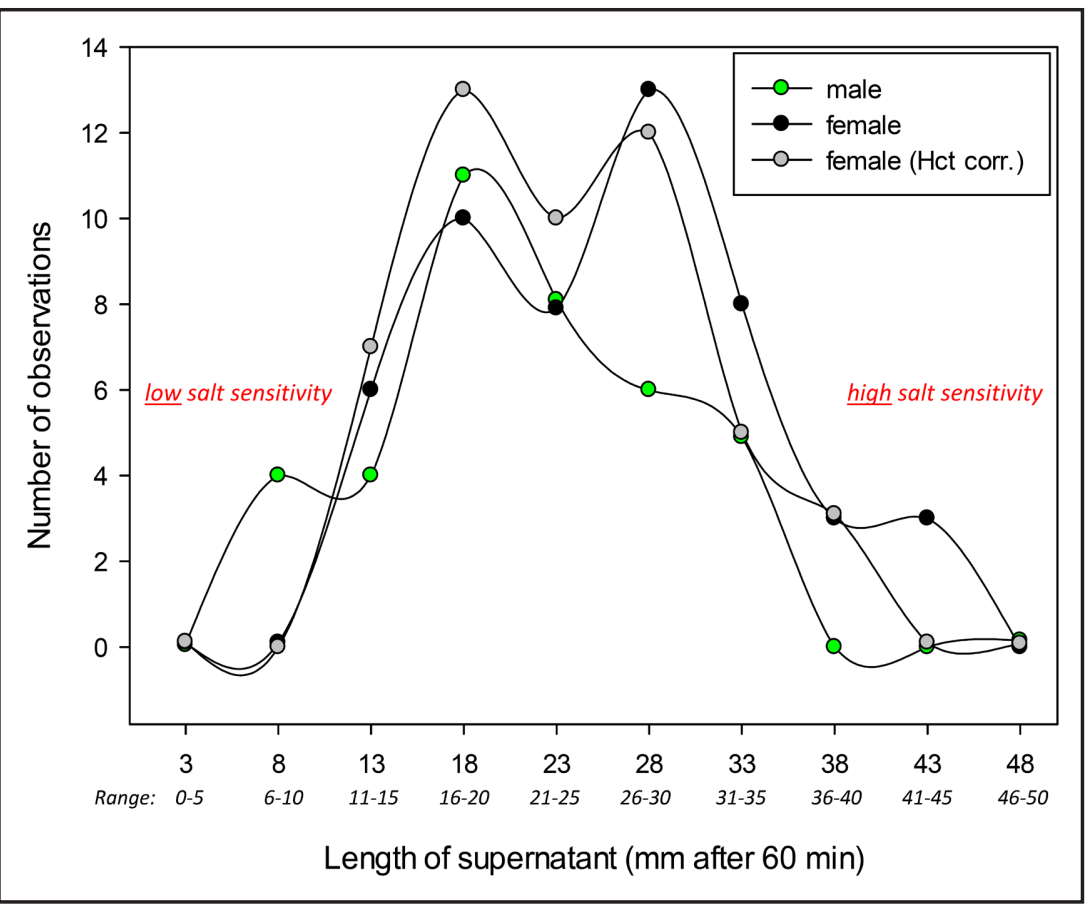

Fig. 4. Histogram curves of the lengths of supernatants obtained from 39 males (green symbols) and 51 females (black symbols without and gray symbols with hematocrit correction).

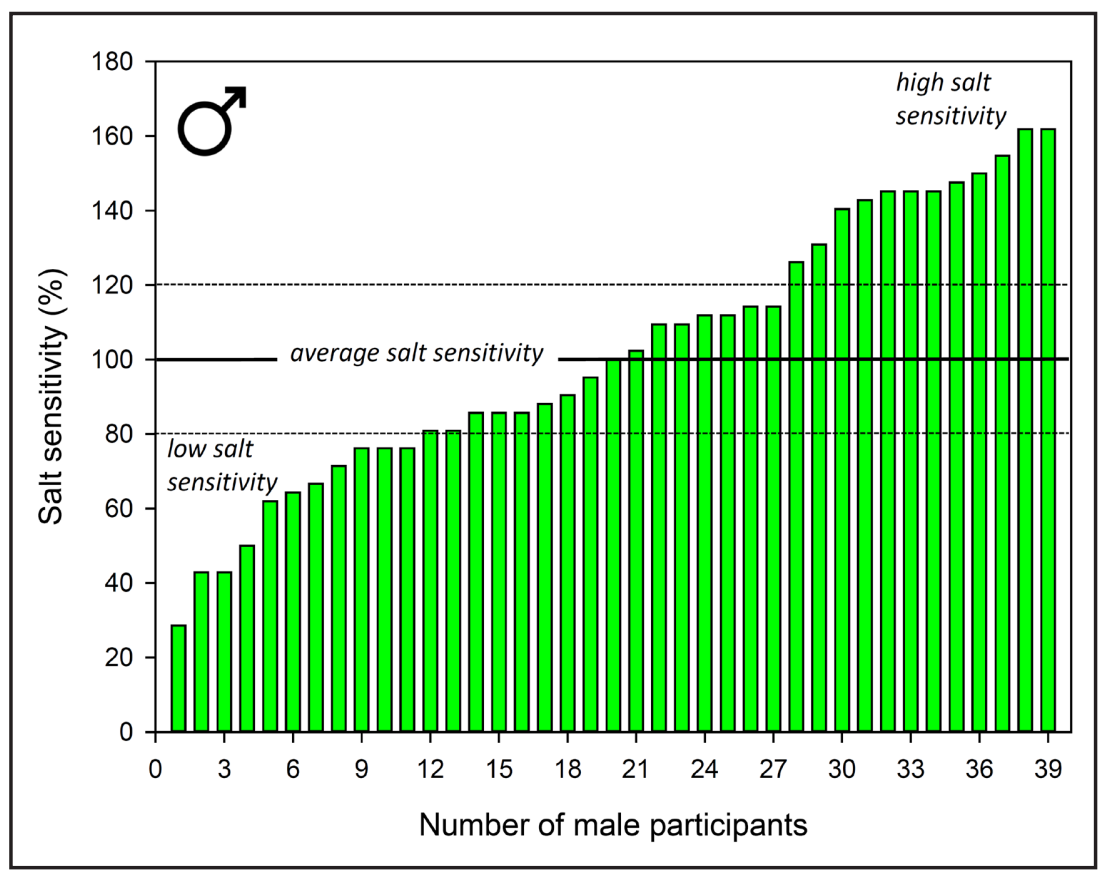

Fig. 5. Histogram showing salt sensitivity (\%) of 39 healthy males (age: $23 \pm 0.5$ years). $100 \%$ indicates mean salt sensitivity of the male cohort. Values between 80 and $120 \%$ are termed "average salt sensitivity". 


\section{Kidney Blood Pressure Research}

Table 1. Technical and biological scatters of the SBT-mini

\begin{tabular}{lcccc}
\hline \multirow{2}{*}{$\begin{array}{l}\text { Parameter } \\
\text { Lengths of supernatants }\end{array}$} & $\begin{array}{c}\text { Single person } \\
\text { Measurements } \\
\text { performed } \\
\text { within 24 hours }\end{array}$ & $\begin{array}{c}\text { Single person } \\
\text { Measurements } \\
\text { performed } \\
\text { within 7 months }\end{array}$ & $\begin{array}{c}\text { Multiple males } \\
\text { Measurements } \\
\text { performed over } \\
\text { two weeks }\end{array}$ & $\begin{array}{c}\text { Multiple females } \\
\text { Measurements } \\
\text { performed over } \\
\text { two weeks }\end{array}$ \\
\hline Mean (mm) & 25.1 & 28.6 & 21.4 & 26.1 \\
Median (mm) & 25.0 & 29.0 & 21.0 & 26.0 \\
Standard deviation (mm) & 1.40 & 4.05 & 7.58 & 8.06 \\
Standard error (mm) & 0.36 & 0.28 & 1.21 & 1.13 \\
Number of measurements & 15 & 211 & 39 & 51 \\
\hline 1st Column indicates the technical scatter (performed in one individual). 2nd column indicates the technical \\
scatter plus the individual scatter of one individual over time. 3 3rd $^{\text {rd }}$ column (males) and 4th column (females) \\
indicate the technical scatter plus the scatter caused by many individuals.
\end{tabular}

large deviation reflects mainly the wide spectrum of SS as shown in figures 5 and 6. Finally, we directly compared data (six individuals) obtained with the original SBT $[12,13]$ and the SBT-mini (figure 7). We found a good correlation between the two tests (correlation coefficient $r^{2}=0.89$ ). Nevertheless, the SBTmini indicates an apparently higher salt sensitivity (in average

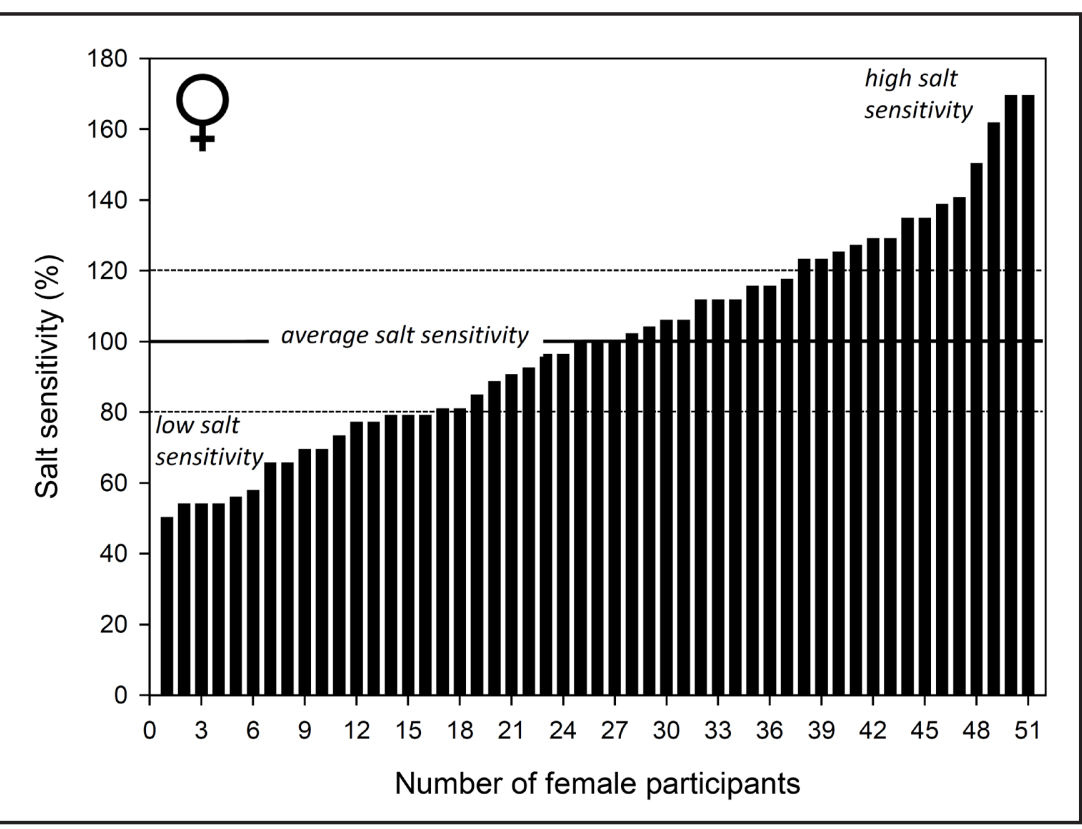

Fig. 6. Histogram showing salt sensitivity (\%) of 51 healthy females (age: $23 \pm 0.5$ years). $100 \%$ indicates mean salt sensitivity of the female cohort. Values between 80 and $120 \%$ are termed "average salt sensitivity". about $+6 \%$ ) as compared to the corresponding values of the original SBT. This difference is negligibly small at high salt sensitivity but appears to increase at very low salt sensitivity. Since the focus is on the detection of high salt sensitivity, this difference should not really matter.

\section{Discussion}

Salt sensitivity of humans is poorly defined. A highly salt (i.e. sodium) sensitive person is likely to respond to salt overload with an increase of arterial blood pressure $[7,17,18]$. It is still unclear what kind of mechanism may be behind the differences in salt sensitivity. Most likely, the kidneys play a major role [19]. The capability of renal sodium excretion determines how long $\mathrm{Na}^{+}$, entering the body after a salty meal, remains in the organism. Since daily salt intake 


\section{Kidney \\ Blood Pressure Research}

in virtually all societies worldwide is usually (too) high (between 2 and 6 grams of $\mathrm{Na}^{+}$), even healthy kidneys reach the limits of their $\mathrm{Na}^{+}$excretion capacity. As a consequence, large quantities of $\mathrm{Na}^{+}$will be transiently deposited in skin [20]. $\mathrm{Na}^{+}$stores will grow with time finally damaging the organism $[21,22]$.

$\mathrm{Na}^{+}$, a small kosmotropic ion with a rather high charge density, is a so-called "water structure maker" (i.e. binds water avidly) [23-25]. Due to the large charge density of $\mathrm{Na}^{+}$, this ion

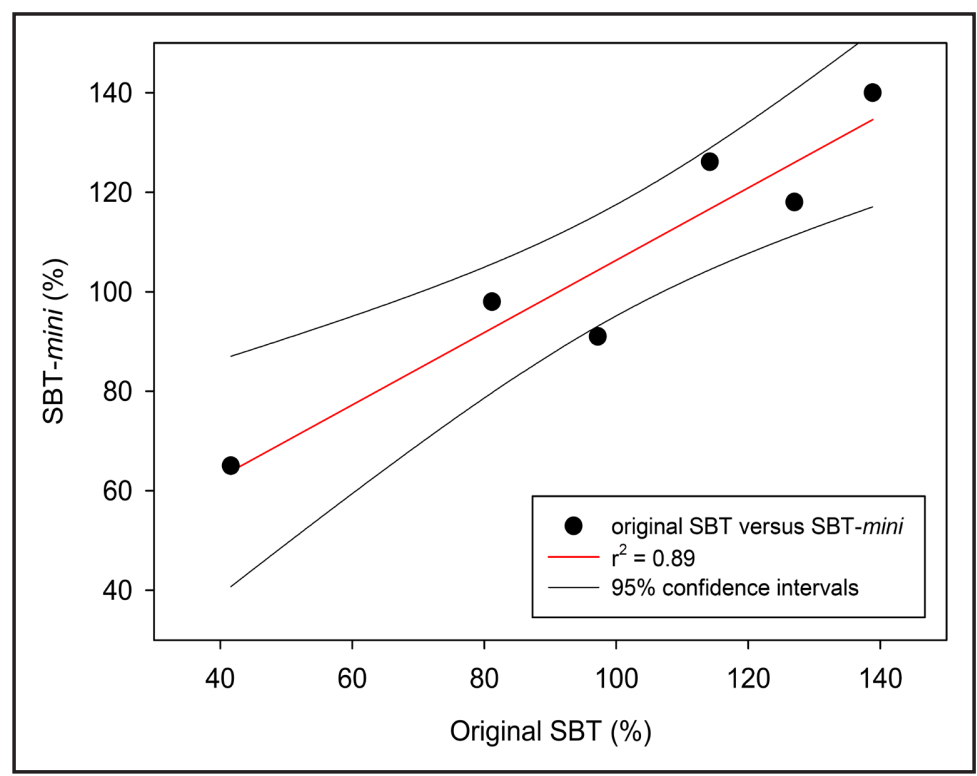

Fig. 7. Direct comparison of data obtained in six individuals (three males/three females; differences in hematocrit were considered). species is preferentially attracted by negatively charged glycocalyx surfaces as endothelium and RBC [16, 26]. Due to its large charge density and its thick water shell, $\mathrm{Na}^{+}$determines the net charge of the glycocalyx. These typical features of this actually 'mousy' ion explain the big impact of $\mathrm{Na}^{+}$on net surface charges, known to influence the zeta potential [15, 27].

Previously, we observed that the erythrocyte glycocalyx 'mirrors' the glycocalyx of the vascular endothelium [13]. Both erythrocyte and endothelial glycocalyces selectively bind $\mathrm{Na}^{+}[16,26]$. Thus, any increase in extracellular $\mathrm{Na}^{+}$(plasma $\mathrm{Na}^{+}$concentration) neutralizes some of the remaining (yet free) negative charges on the erythrocyte/endothelial surface. This will influence the zeta potential. Based on this concept, the so-called salt blood test (SBT) was developed three years ago [12]. Applying the SBT to healthy volunteers, two subgroups could be identified, a low and a high salt sensitive group. In the meantime, two clinical studies were performed. One study showed that patients with manifest hypertension are more frequently found in the highly salt sensitive subgroup [14]. Another study, carried out with the blood of dialysis patients, showed that the RBC glycocalyx is improved after acute hemodialysis, i.e. salt sensitivity decreases or, in other words, the $\mathrm{Na}^{+}$buffering power of the glycocalyx recovers by a 4-hour hemodialysis of patients suffering from chronic kidney disease [28].

Aiming to reach not only patients under professional medical care but also to address healthy people without specific medical education we set out trying to make the SBT easier to use. The SBT-mini as described here is the result of these efforts. In comparison to the original SBT the SBT-mini has advantages and disadvantages. The advantages are as follows: (i) Minimal equipment and only a few steps are sufficient to quantify salt sensitivity. (ii) Only $50 \mu \mathrm{l}$ of capillary blood is enough to perform the test. A potential disadvantage of the SBT-mini is the fact that the individual hematocrit influences the result. This is only critical when larger hematocrit fluctuations are to be expected, e.g. in anemic/hyperemic patients. Even under these conditions, the SBT-mini can still be performed but one has to be aware that the result of such a measurement includes both, RBC quantity (hematocrit) and the RBC quality ( $\mathrm{Na}^{+}$buffer capacity). If these two variables ought to be separated, then the hematocrit must be measured. The significant difference in hematocrit between males and 


\section{Kidney Blood Pressure Research}

Kidney Blood Press Res 2016;41:355-364

DOI: $10.1159 / 000443438$

Published online: June 16, 2016

(C) 2016 The Author(s). Published by S. Karger AG, Base www.karger.com/kbr

females was considered when the SBT-mini was developed. We offer two standard values, one for females and one for males. Using these two standards the influence of sex differences in the hematocrit is minimized.

The results of the original SBT compare well with the data obtained with the SBT-mini. When the hematocrit differences between males and females is taken into account we do not find a significant sex difference in SS. This agrees with the results of the original SBT [12]. Furthermore, the original SBT showed that $28 \%$ of the (male/female) study participants (61 healthy young persons, mean age 23 years) were highly salt sensitive (SS $>20 \%$ of average value). This matches very well with the data of the present study performed on a comparable cohort (90 healthy young people, mean age 23 years) where $31 \%$ of the male cohort and 28 $\%$ of the female cohort were found highly salt sensitive.

In comparison to the original SBT published previously the SBT-mini does not need any centrifugation and $\mathrm{RBC}$ washing procedures but uses a mixture of whole capillary blood with one single 'smart' $\mathrm{Na}^{+}$cocktail. It contains a low $\mathrm{Na}^{+}$concentration that, on the one hand, is high enough to prevent any hemolysis (osmolarity: about $200 \mathrm{mOsmol} / \mathrm{l}$ ) and, on the other hand, is low enough to maintain a sufficient moiety of free negative RBC surface charges. RBC sedimentation rate depends crucially on this well-defined balance between $\mathrm{Na}^{+}$and the (still unshielded) free negative charges. We tested many different $\mathrm{Na}^{+}$concentrations (some curves are shown in figure 2) and finally chose the most suitable one. 'Most suitable' means that the sedimentation rate (indirectly representing the zeta potential) measured after 60 minutes is well readable from the test tube so that even extreme values (i.e. extremely low and high salt sensitive donor blood) can be measured. In contrast to the original SBT in which $\mathrm{Ca}^{2+}$ was removed by the washing procedures we used EDTA to chelate $\mathrm{Ca}^{2+}$. This is necessary because $\mathrm{Ca}^{2+}$ is known to strongly interfere with charged surfaces and thus would mask the $\mathrm{Na}^{+}$associated changes of the zeta potential [26]. Finally, we modified the dextrane concentration. Dextrane is necessary to render RBC surfaces 'sticky' facilitating RBC aggregation. As described previously, dextrane sticks to the very surface of RBC [15, 27]. The right molecular size is quite critical since the dextrane molecules will 'hide' in the glycocalyx and RBC aggregate when RBC surfaces come (physically) close to each other (few nanometers). The distance depends upon the 'quality' of the glycocalyx. A poor glycocalyx has a reduced number of electrical charges and thus RBC aggregate more easily. Dextrane facilitates this tendency for aggregation and makes this phenomenon measurable (sedimentation rate).

Similar as already shown with using the original SBT, the SBT-mini discloses a doublepeak histogram in the healthy population indicating that there may exist two subgroups, a low salt sensitive and a high salt sensitive group. It seems to be more pronounced in women as in men. SS values in the range of 80 to $120 \%$ were termed 'average SS' indicating that this is about the range (about 3 times the standard error) expected for healthy persons (including all types of SS). The double-peak histograms cannot simply be explained by genetic predisposition [29] but can be also due to distinct differences in life style of the individuals [30]. For example, application of polyphenolic compounds in vitro [12] and in vivo [16] can influence SS. Also elevated concentrations of plasma sodium can gradually destroy the charged surface layers of endothelium and RBC as recently demonstrated [8, 31, 32]. Furthermore, SS changes in inflammatory diseases/atherothrombotic processes are likely to occur since endothelial surface layers are known to lose their negative charges under such conditions [33-35]. It can be concluded that any changes in $\mathrm{Na}^{+}$buffer capacity of the RBC glycocalyx will be reported by the SBT-mini. This may happen in many different diseases such as cancer, immune and infectious diseases. Nevertheless, such changes do indicate an altered SS of these individuals.

The concept of the SBT-mini is clearly different in comparison to the classical measurement of erythrocyte sedimentation rate (ESR). RBC sedimentation as measured by the SBT-mini depends mainly on the $\mathrm{Na}^{+}$-specific properties of the RBC surface layer (blood 


\section{Kidney \\ Blood Pressure Research}

Oberleithner/Wilhelmi: SBT-mini

is diluted by $\mathrm{Na}^{+}$cocktail, $\mathrm{Ca}^{2+}$ has been chelated by EDTA and $\mathrm{K}^{+}$is known for its negligible interference with the RBC glycocalyx [16]) while ESR performed in whole blood depends mainly on the functional characteristics of plasma proteins and their inference with RBC.

\section{Conclusion}

The SBT-mini could be suitable for evaluating salt sensitivity in humans. With a technical error of $5 \%$, easy handling and good reliability the SBT-mini should promise wide-spread use. Since SS is not a genetically-fixed but a rather variable value, the test could be useful for evaluating the individual's response to any preventive actions (e.g. low-salt diet, life style changes) and/or to drug treatment in cardiovascular diseases.

\section{Disclosure Statement}

The authors of this manuscript state that they do not have any conflict of interests and nothing to disclose.

\section{Acknowledgements}

Work was supported by Cells-in-Motion Cluster of Excellence (EXC 1003-CIM). The experimental protocol was approved by the local Ethics Committee (Ärztekammer WestfalenLippe, approval number 2012-029-f-S). We thank Barbara Windoffer and Sergei Handel for their excellent laboratory work. Patent applications for the salt blood test have been filed (Publication Nos. EP2926132 and US 2015/0308939).

\section{References}

1 Walkowska A, Kuczeriszka M, Sadowski J, Olszynski KH, Dobrowolski L, Cervenka L, Hammock BD, Kompanowska-Jezierska E: High salt intake increases blood pressure in normal rats: putative role of 20HETE and no evidence on changes in renal vascular reactivity. Kidney Blood Press Res 2015;40:323-334.

2 Weinberger MH: Salt and blood pressure. Curr Opin Cardiol 2000;15:254-257.

3 Mozaffarian D, Fahimi S, Singh GM, Micha R, Khatibzadeh S, Engell RE, Lim S, Danaei G, Ezzati M, Powles J: Global sodium consumption and death from cardiovascular causes. N Engl J Med 2014;371:624-634.

4 Meneton P, Jeunemaitre X, de Wardener HE, MacGregor GA: Links between dietary salt intake, renal salt handling, blood pressure, and cardiovascular diseases. Physiol Rev 2005;85:679-715.

$5 \quad$ Ritz E: Salt - friend or foe? Nephrol Dial Transplant 2006;21:2052-2056.

6 Funder JW: Primary aldosteronism and salt. Pflügers Arch - Eur J Physiol 2015;467:587-594.

7 He FJ, MacGregor GA: Salt and sugar: their effects on blood pressure. Pflügers Arch - Eur J Physiol 2015;467:577-586.

8 Oberleithner H: Vascular endothelium: a vulnerable transit zone for merciless sodium. Nephrol Dial Transplant 2013;29:240-246.

9 De La Sierra A, Giner V, Bragulat E, Coca A: Lack of correlation between two methods for the assessment of salt sensitivity in essential hypertension. J Hum Hypertens 2002;16:255-260.

10 Nichols J, Elijovich F, Laffer CL: Lack of validation of a same-day outpatient protocol for determination of salt sensitivity of blood pressure. Hypertension 2012;59:390-394.

11 Felder RA, White MJ, Williams SM, Jose PA: Diagnostic tools for hypertension and salt sensitivity testing. Curr Opin Nephrol Hypertens 2013;22:65-76. 


\section{Kidney \\ Blood Pressure Research}

12 Oberleithner H, Wilhelmi M: Determination of erythrocyte sodium sensitivity in man. Pflugers Arch - Eur J Physiol 2013;465:1459-1466.

13 Oberleithner H: Vascular endothelium leaves fingerprints on the surface of erythrocytes. Pflügers Arch Eur J Physiol 2013; 465:1451-1458.

14 Oberleithner H, Wilhelmi M: Vascular glycocalyx sodium store - determinant of salt sensitivity. Blood Purif 2015;39:7-10.

15 Jan KM, Chien S: Influence of the ionic composition of fluid medium on red cell aggregation. J Gen Physiol 1973;61:655-668.

16 Oberleithner H: Sodium selective erythrocyte glycocalyx and salt sensitivity in man. Pflügers Arch - Eur J Physiol 2014;467:1319-1325

17 Ritz E: Salt and hypertension. Nephrology (Carlton ) 2010;15:S49-52.

18 Weinberger MH: Salt sensitivity of blood pressure in humans. Hypertension 1996;27:481-490.

19 Guyton AC: Blood pressure control--special role of the kidneys and body fluids. Science 1991;252:18131816.

20 Titze J, Lang R, Ilies C, Schwind KH, Kirsch KA, Dietsch P, Luft FC, Hilgers KF: Osmotically inactive skin Na+ storage in rats. Am J Physiol Renal Physiol 2003;285:F1108-F1117.

21 Kleinewietfeld M, Manzel A, Titze J, Kvakan H, Yosef N, Linker RA, Muller DN, Hafler DA: Sodium chloride drives autoimmune disease by the induction of pathogenic T17 cells. Nature 2013;496:518-522.

22 McMaster WG, Kirabo A, Madhur MS, Harrison DG: Inflammation, immunity, and hypertensive end-organ damage. Circ Res 2015;116:1022-1033.

23 Hribar B, Southall NT, Vlachy V, Dill KA: How ions affect the structure of water. J Am Chem Soc 2002;124:12302-12311.

24 Collins KD: Charge density-dependent strength of hydration and biological structure. Biophys J 1997;72:65-76.

25 Kiriukhin MY, Collins KD: Dynamic hydration numbers for biologically important ions. Biophys Chem 2002;99:155-168.

26 Bevan JA: Flow regulation of vascular tone. Its sensitivity to changes in sodium and calcium. Hypertension 1993;22:273-281.

27 Jan KM, Chien S: Role of surface electric charge in red blood cell interactions. J Gen Physiol 1973;61:638654.

28 Kliche K, Gerth U, Pavenstadt H, Oberleithner H: Recharging red blood cell surface by hemodialysis. Cell Physiol Biochem 2015;35:1107-1115.

29 Sanada H, Jones JE, Jose PA: Genetics of salt-sensitive hypertension. Curr Hypertens Rep 2011;13:55-66.

30 Hoffmann IS, Alfieri AB, Cubeddu LX: Effects of lifestyle changes and metformin on salt sensitivity and nitric oxide metabolism in obese salt-sensitive Hispanics. J Hum Hypertens 2007;21:571-578.

31 Oberleithner H, Walte M, Kusche-Vihrog K: Sodium renders endothelial cells sticky for red blood cells. Front Physiol 2015;6:188.

32 Kusche-Vihrog K, Schmitz B, Brand E: Salt controls endothelial and vascular phenotype. Pflügers Arch - Eur J Physiol 2015;467:499-512.

33 Noble MI, Drake-Holland AJ, Vink H: Hypothesis: arterial glycocalyx dysfunction is the first step in the atherothrombotic process. QJM 2008;101:513-518.

34 Padberg JS, Wiesinger A, Di Marco GS, Reuter S, Grabner A, Kentrup D, Lukasz A, Oberleithner H, Pavenstadt $\mathrm{H}$, Brand M, Kumpers P: Damage of the endothelial glycocalyx in chronic kidney disease. Atherosclerosis 2014;234:335-343.

35 Wiesinger A, Peters W, Chappell D, Kentrup D, Reuter S, Pavenstadt H, Oberleithner H, Kumpers P: Nanomechanics of the endothelial glycocalyx in experimental sepsis. PLoS One 2013;8:e80905. 